\title{
High expression of DDR1 is associated with the poor prognosis in Chinese patients with pancreatic ductal adenocarcinoma
}

Yanmiao Huo ${ }^{\dagger}$, Minwei Yang ${ }^{\dagger}$, Wei Liư ${ }^{\dagger}$ Jianyu Yang ${ }^{\dagger}$, Xueliang Fu, Dejun Liu, Jiao Li, Junfeng Zhang, Rong Hua ${ }^{*}$ and Yongwei Sun

\begin{abstract}
Background: Discoidin domain receptors 1 (DDR1), a subtype of DDRs, has been reported as a critical modulator of cellular morphogenesis, differentiation, migration and invasion.

Methods and results: In this study, we investigated the expression of DDR1 and its clinical association in Chinese patients with pancreatic ductal adenocarcinoma (PDAC). Across a cohort of 30 patients, we examined DDR1 expression in paired PDAC and corresponding adjacent non-tumor tissues by real-time quantitative PCR (RT-qPCR), or western blotting. DDR1 expression is significantly higher in PDAC, as compared to normal adjacent tissue, confirming results from the Oncomine databases. We validated DDR1 expression by immunohistochemistry across a non-overlapping cohort of 205 PDAC specimens. Kaplan-Meier survival curves indicate that increased expression of DDR1 is associated with a poor prognosis in PDAC patients $(P=0.013)$. Multivariate Cox regression analysis identified DDR1 expression, age, $\mathrm{N}$ classification and liver metastasis as independent prognostic factors in PDAC.
\end{abstract} Conclusions: This study demonstrated that DDR1 can well serve as a novel prognostic biomarker in PDAC.

Keywords: DDR1, Pancreatic ductal adenocarcinoma, Prognosis

\section{Introduction}

Pancreatic ductal adenocarcinoma (PDAC) is a devastating disease and the fourth most common disease-related mortality worldwide $[1,2]$. The 5 -year survival is approximately $6 \%$, and only $20 \%$ of patients present with resectable disease $[3,4]$. Few PDAC cases (less than $10 \%$ ) are diagnosed at early stages [5], largely due to the absence of specific symptoms, and as such patients often present with advanced stage disease. Hence, the identification of novel prognostic indicators has become a major topic of interest to the PDAC research community at large.

The discoidin domain receptor 1 (DDR1) belongs to a subfamily of Receptor tyrosine kinases (RTKs) characterized by the presence of an extracellular discoidin homology domain that function to modulates cell proliferation and differentiation. DDRs are non-integrin collagen receptors

\footnotetext{
*Correspondence: lordhuarong@sohu.com; syw0616@126.com

${ }^{\dagger}$ Equal contributors

Department of Biliary-Pancreatic Surgery, Ren Ji Hospital, School of Medicine, Shanghai Jiao Tong University, 1630 Dongfang Road, Shanghai 200127, P.R. China
}

(c) 2015 Huo et al. Open Access This article is distributed under the terms of the Creative Commons Attribution 4.0 International License (http://creativecommons.org/licenses/by/4.0/), which permits unrestricted use, distribution, and reproduction in any medium, provided you give appropriate credit to the original author(s) and the source, provide a link to the Creative Commons license, and indicate if changes were made. The Creative Commons Public Domain Dedication waiver (http://creativecommons.org/publicdomain/zero/1.0/) applies to the data made available in this article, unless otherwise stated.

composed of two types, DDR1 and DDR2, independently activated by receptor-specific collagens binding at the discoidin domain [6]. DDR1 can be alternatively spliced into five isoforms (DDR1a-e). Recently, studies have suggested DDR1 participates in several critical cell processes including: adhesion, migration, proliferation, and invasion [7-9]. Moreover, expression of DDR1 is known to be dysregulated in multiple human cancers such as lung, breast, hepatic, and ovary cancers, suggesting a previously unappreciated role of DDR1 in tumor formation and progression [10-13]. In PDACs, however, the role of DDR1 remains to be uncharacterized.

In this retrospective study, we examined the expression pattern of DDR1 at the mRNA and protein level and explored the relationship of DDR1 expression with clinicopathologic parameters, including overall survival. We found that the expression of DDR1 was associated with poor prognosis of Chinese PDAC patients. 


\section{Materials and methods Ethics statement}

This research was approved by the Ethics Committee of Ren Ji hospital, School of Medicine, Shanghai Jiao Tong University, and written informed consent was obtained from each patient involved in this study.

\section{Patients and tissue specimens}

We retrospectively analyzed clinical and pathological characteristics of 205 patients who had resectable infiltrating PDAC and underwent surgical resection at the Biliary-Pancreatic Department of Ren Ji Hospital, School of Medicine, Shanghai Jiao Tong University, China, from January 2002 to June 2014 (see Table 1). Patients who had history of other solid tumors, received preoperative chemotherapy, radiotherapy or other anticancer therapies were excluded from this study. Standard pancreatectomy was undergone with lymph node dissection in patients who had no evidence of distant organ metastasis. Routine chemotherapy with gemcitabine had been given to all patients after operation, but no radiation treatment was done in any of the patients included in our study [14]. An additional 30 fresh frozen tissues of PDAC and corresponding adjacent non-tumor tissues (located more than $2 \mathrm{~cm}$ apart from the tumor tissue in each case) were also obtained from the same department [15]. The diagnosis was confirmed by two clinical pathologists.

\section{RNA extraction and real-time quantitative PCR (RT-qPCR)}

Total RNA from primary tumor and adjacent non-tumor tissue samples was isolated with Trizol reagent (Takara, Japan), and reversely transcribed through PrimeScript RTqPCR kit (Takara, Japan) according to the manufacturer's instructions [16]. RT-qPCR was performed using a 7500 RT-qPCR system (Applied Biosystems, Inc. USA) with a 15- $\mu \mathrm{l}$ PCR mix containing $0.5 \mu \mathrm{l}$ of cDNA, $7.5 \mu \mathrm{l}$ of $2 *$ SYBR Green master mix (Invitrogen, Carlsbad, California, USA), and $200 \mathrm{nM}$ of the appropriate primers (Invitrogen, Carlsbad, California, USA). Primer sequences set for DDR1 detection were as follows: forward primer: 5'GCGTCTGTCTGCGGGTAGAG-3', reverse primer: 5'ACGGCCTCAGATAAATACATTGTCT-3'. The relative levels of mRNA expression were calculated based on the difference between amplification of DDR1 and $\beta$-actin RNA (forward: 5'-ACTCGTCATACTCCTGCT-3', reverse: 5'- GAAACTACCTTCAACTCC-3') using the $2^{-\Delta \Delta \mathrm{ct}}$ method [17]. To minimize technical (run-to-run) variation between the samples, all samples were analyze $d$ in the same run for both target genes and reference genes. All experiments were performed three times with three technical replicates.
Table 1 Association between DDR1 expression and clinicopathologic features in patients with PDAC

\begin{tabular}{|c|c|c|c|c|}
\hline \multirow{3}{*}{ Characteristics } & \multirow[b]{3}{*}{ Total } & \multicolumn{2}{|c|}{ DDR1 Expression } & \multirow{3}{*}{$\begin{array}{l}P \text { value } \\
\text { (x2 test) }\end{array}$} \\
\hline & & High & Low & \\
\hline & & $(n=126)$ & $(n=79)$ & \\
\hline \multicolumn{5}{|l|}{ Age (years) } \\
\hline Mean (years) & 65.19 & 66.82 & 62.69 & 0.374 \\
\hline$<65$ & 107 & 65 & 42 & 0.826 \\
\hline$\geq 65$ & 98 & 61 & 37 & \\
\hline Gender & & & & 0.891 \\
\hline Male & 117 & 74 & 43 & \\
\hline Female & 88 & 52 & 36 & \\
\hline Tumor location & & & & 0.273 \\
\hline Head & 139 & 89 & 50 & \\
\hline Body/tail & 66 & 37 & 29 & \\
\hline Size & & & & 0.551 \\
\hline$\leq 2 \mathrm{~cm}$ & 27 & 18 & 9 & \\
\hline$>2 \mathrm{~cm}$ & 178 & 108 & 70 & \\
\hline Tumor differentiation & & & & 0.054 \\
\hline Well & 11 & 10 & 1 & \\
\hline Moderate/poor & 194 & 116 & 78 & \\
\hline T classification & & & & 0.697 \\
\hline $\mathrm{T} 1$ & 11 & 8 & 3 & \\
\hline $\mathrm{T} 2$ & 31 & 18 & 13 & \\
\hline T3 & 125 & 73 & 52 & \\
\hline $\mathrm{T} 4$ & 38 & 20 & 18 & \\
\hline N classification & & & & 0.858 \\
\hline Absent & 136 & 83 & 53 & \\
\hline Present & 69 & 43 & 26 & \\
\hline AJCC stage & & & & 0.949 \\
\hline Stage I & 38 & 20 & 18 & \\
\hline Stage II & 135 & 73 & 62 & \\
\hline Stage III & 21 & 10 & 11 & \\
\hline Stage IV & 14 & 7 & 7 & \\
\hline Liver metastasis & & & & 0.560 \\
\hline Absent & 191 & 118 & 73 & \\
\hline Present & 14 & 8 & 6 & \\
\hline Vascular invasion & & & & 0.801 \\
\hline Absent & 177 & 109 & 68 & \\
\hline Present & 28 & 17 & 11 & \\
\hline Vital status & & & & 0.912 \\
\hline Dead & 172 & 106 & 66 & \\
\hline Alive & 33 & 20 & 13 & \\
\hline
\end{tabular}

AJCC staging is according to the $7^{\text {th }}$ edition of the American Joint Committee on Cancer (AJCC) staging system [29] 

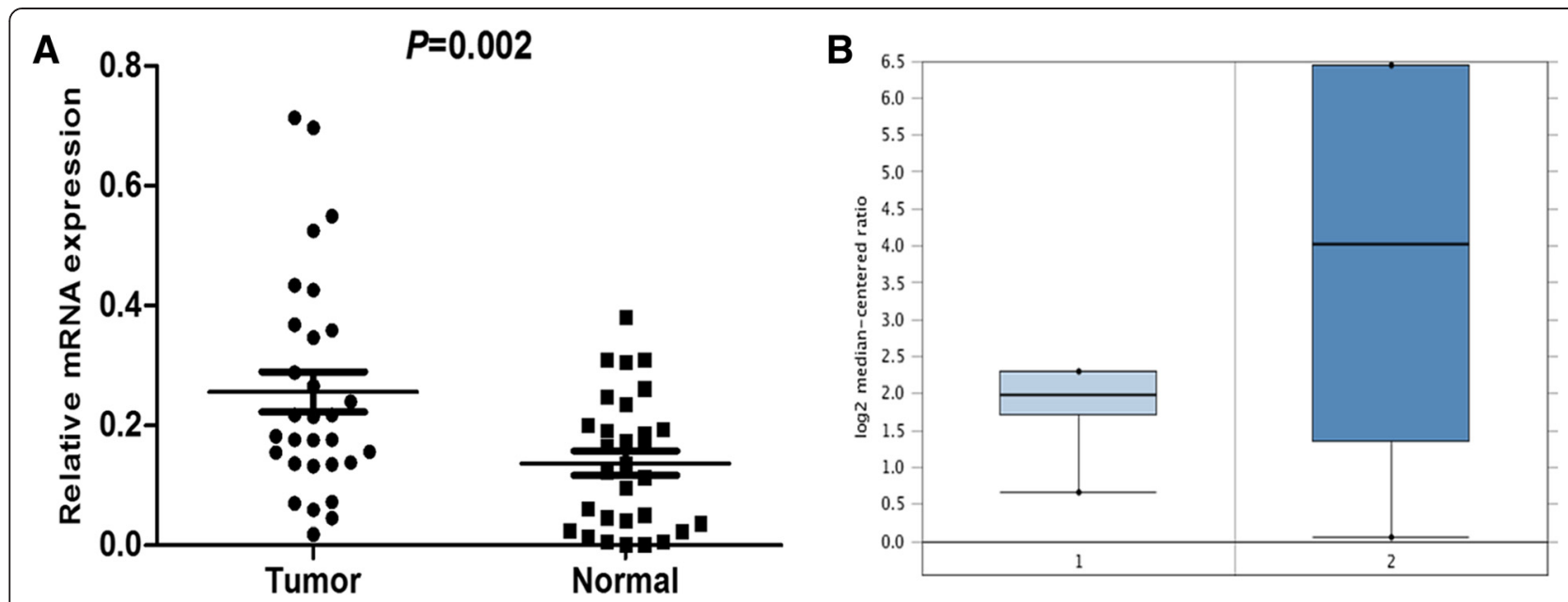

Fig. 1 DDR1 expression is increased in PDAC at mRNA level. a increased DDR1 mRNA expression in 30 matched tumor (T) and non-tumor tissue (N) was detected by real-time quantitative PCR. b DDR1 expression in Buchholz pancreas grouped by normal pancreatic duct (1) and PDAC (2)

\section{Western blotting analysis}

Western blotting was performed as previously described [18]. The DDR1 antibody was purchased from Proteintech Inc. and species-specific secondary antibody was purchased from Cell Signaling, Beverly, MA. Bound secondary antibodies were detected by Odyssey imaging system (LI-COR Biosciences, Lincoln, NE).

\section{Tissue microarray (TMA) construction}

Tissue microarrays (TMA) were constructed using diameter of $1.5-\mathrm{mm}$ cores including 205 cases of matched tumor and non-tumor tissues specimens. After screening and marking representative spots of tissues, the tissues were punched out and squeezed into the paraffin array blocks.

\section{Immunohistochemical (IHC) staining and scoring}

Immunohistochemical staining was performed on a tissue microarray (TMA) containing 205 paired PDAC samples as previous described [3]. Scoring was calculated according to the sum of the percentage of positively stained tumor cells: 0-5 \% scored 0; $6 \%-35 \%$ scored 1; $36 \%-70 \%$ scored 2; more than $70 \%$ scored 3 and the staining intensity: no staining scored 0 , weakly staining scored 1 , moderately staining scored 2 and strongly staining scored 3, respectively. The final score was designated as low or high expression group using the percentage of cells staining positive multiplied by the staining intensity as follows: "-" for a score of $0-1$, " + " for a score of $2-3$, " ++ " for a score of $4-6$ and "+++" for a score of $>6$; low expression was defined as a total score $<4$ and high expression with a total score $\geq 4$.

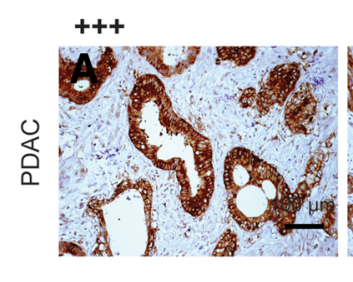

E

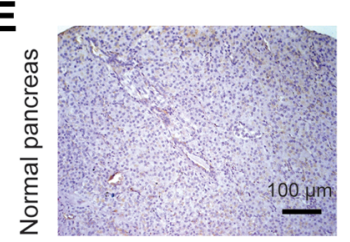

$\mathbf{F}$
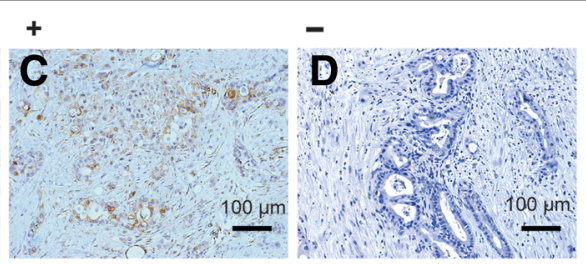

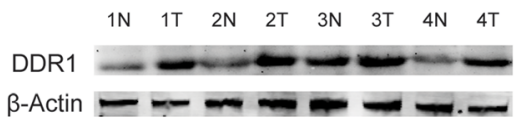

Fig. 2 DDR1 protein expression in PDAC tissue samples. a-e Immunohistochemical representative images of DDR1 expression in PDAC compared with surrounding noncancerous pancreas (SNP) analyzed by immunohistochemisty; a PDAC, scored as (+++); b PDAC, scored as (++); c PDAC, scored as (+); $\mathbf{d}$ PDAC, scored as (-); e Normal pancreas, scored as (-). $\mathbf{f}$ Western blots of DDR1 expression in four pairs of PDAC patients 


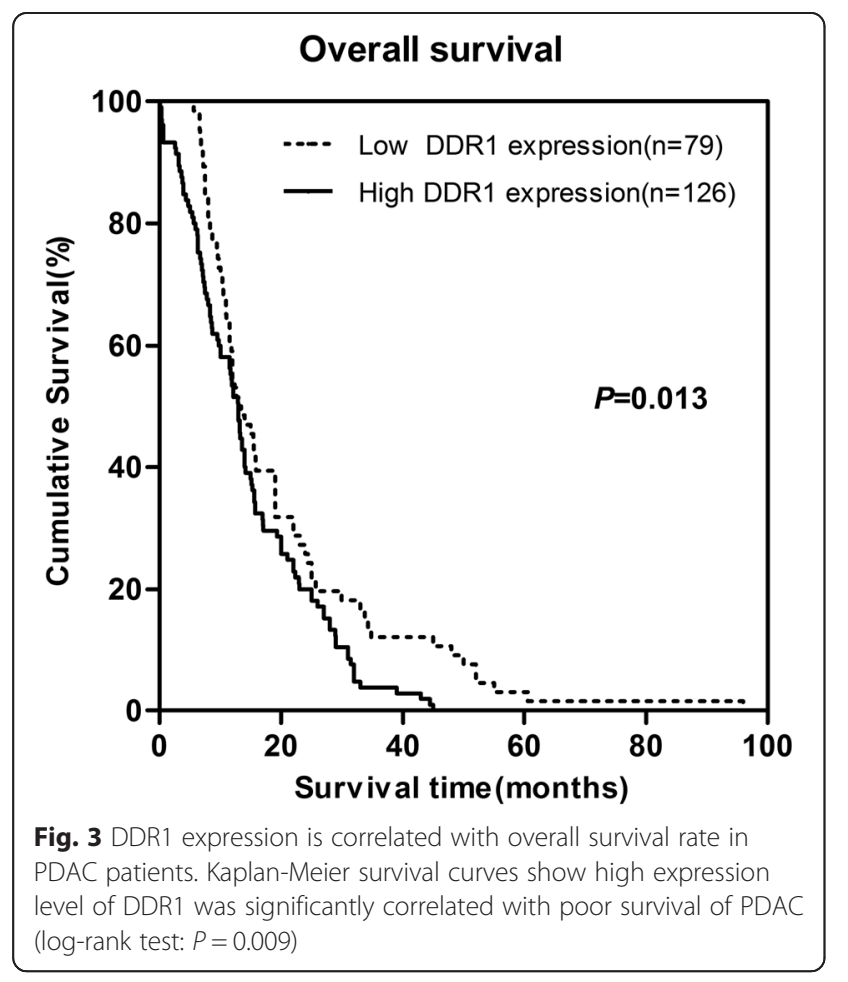

These scores were determined independently by two senior pathologists. The scoring by the pathologists was done in a blinded manner.

\section{Follow-up}

The postoperative follow-up included clinical and laboratory examinations. OS, a measure of prognosis, was defined as the time from the date of surgery to the date of death or the last follow-up examination.

\section{Statistical analysis}

All statistical analyses were performed using SPSS 19.0 (SPSS Inc., Chicago, IL, USA) and GraphPad Prism 5 (San Diego, CA) software. DDR1 mRNA in the tumor and adjacent non-tumor tissue samples were compared using a paired-samples $t$ test. The Chi-square test and Fisher's exact probability method was used to analyze the relationship between DDR1 expression and clinicopathological characteristics. Survival curves were evaluated using the Kaplan-Meier method, and differences between survival curves were tested by the log-rank test. Cox proportional hazards regression model was used to examine univariate and multivariate hazard ratios for the study variables. Only significantly different variables in univariate analysis including DDR1 expression level, Age, N classification, Liver metastasis were entered into the next multivariate analysis [19]. A two-sided $P$-value $<0.05$ was considered statistically significant.

\section{Results}

DDR1 is transcriptionally upregulated in PDAC

We evaluated the transcript levels of DDR1, by RTqPCR, using 30 pairs of specimens from PDAC patients. The DDR1 mRNA levels were significantly increased in 19/30 PDAC tissue samples compared with the matched adjacent non-tumor tissue samples $(P=0.002$, Fig. 1a). Additionally, we analyzed a microarray datasets from Oncomine databases showed in Fig. 1b [20] and found mRNA expression levels of DDR1 was consistent with our data.

\section{DDR1 expression at protein level in PDAC}

Based on the IHC scoring criterions established (see methods), 126 of 205 (61.5\%) PDACs specimens showed high DDR1 expression (DDR1 ++ or DDR1 +++), whereas
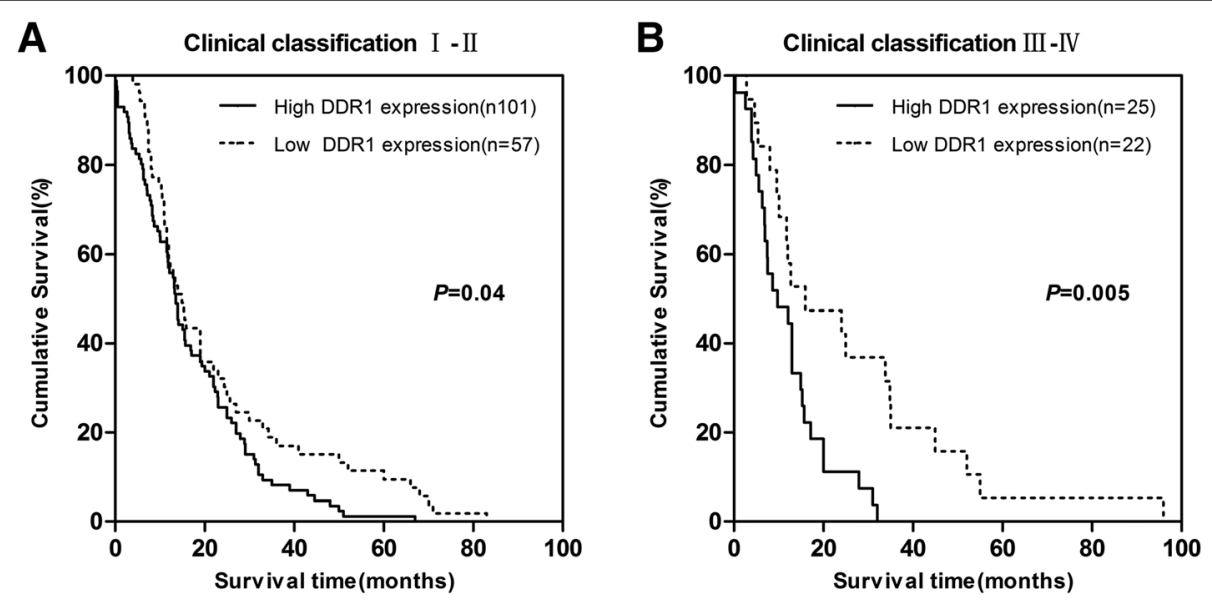

Fig. 4 Comparisons of overall survival between DDR1 high expression and DDR1 low expression in early clinical stage (I-II) cohort and in advanced clinical stage (III-IV) cohort. P-values were calculated by log-rank test 

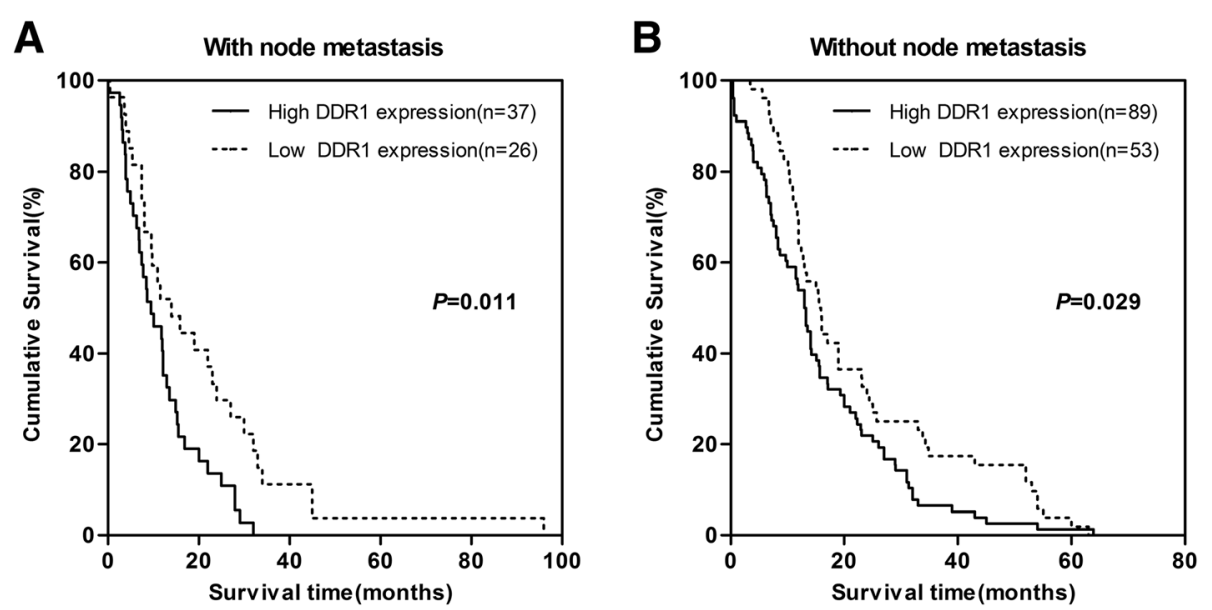

Fig. 5 Comparisons of overall survival between DDR1 high expression and DDR1 low expression in patients with or without lymph node metastasis. $P$-values were calculated by log-rank test

the remaining 79 cases (38.5 \%) displayed low DDR1 expression (DDR1- or DDR1 +) (Fig. 2 a-e). We valdated the protein overexpression across 4 pairs of resected representative specimens (tumor tissues and matched adjacent nontumor tissues) from PDAC patients using Western blotting analysis. Consistent with the RT-qPCR results, an increase in DDR1 expression was observed in all of the 4 PDAC tissues compared with the matched adjacent non-tumor tissues (Fig. 2f).

\section{Correlation of DDR1 expression with clinicopathological features of PDAC}

To determine the relationship between DDR1 expressions with the clinicopathological features of PDAC, the IHC staining of DDR1 levels were statistically evaluated by the Chi-square tests. The clinicopathologic parameters in PDAC included: age, gender, clinical stage, liver metastasis, vascular invasion and differentiation status.
As shown in Table 1, no significant differences were found between DDR1 expression and any other parameters.

\section{Correlation of DDR1 expression and prognosis in PDAC patients}

The patients' survival analysis was evaluated by Kaplan-Meier analysis and log-rank test. As shown in Fig. 3, there exists a negative correlation between DDR1 expression and overall survival $(P=0.013)$. The association between DDR1 expression and overall survival in PDAC patients was also evaluated with regards to clinical stages, status of lymphatic metastasis and vascular invasion (Fig. 4, 5 and 6). The overall survival time was significantly different between patients with low and high DDR1 expression $(P<0.05)$, with the low DDR1 group having a longer overall survival independent of the clinical stage, status of lymphatic metastasis and vascular invasion.
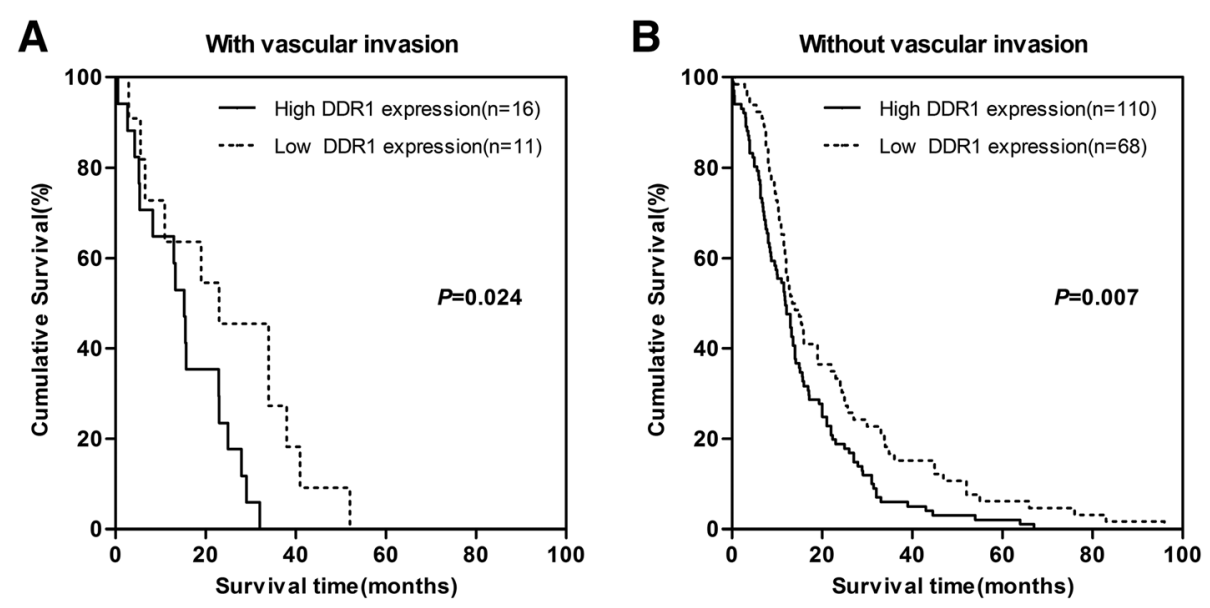

Fig. 6 Comparisons of overall survival between DDR1 high expression and DDR1 low expression in patients with or without vascular invasion. $P$-values were calculated by log-rank test 


\section{Univariate and multivariate analyses}

Univariate and multivariate analyses were performed to compare the impact of DDR1 expression and other clinical and pathological parameters across our cohort (Table 2). Based on results of the univariate analyses, the age, DDR1 expression, $\mathrm{N}$ classification and Liver metastasis were significantly associated with overall survival. Furthermore, all these 4 factors were included in a multivariate Cox regression analysis to adjust for the effects of the covariates and were confirmed as independent prognostic factors.

\section{Discussion}

Previously studies have identified DDR1 is overexpressed in various human invasive tumors including lung, breast, hepatic, and ovary cancers, highlighting its possible role in tumor initiation, maintenance or progression [10-13]. In the present study, DDR1 expression and its association with clinicopathological features including prognosis were investigated across a cohort of Chinese PDAC patients.

We determined that DDR1 expression was increased in Chinese PDAC patients at both the mRNA and protein level. These findings are supported by non-overlapping data from an Oncomine database, which highlights the same trends in PDAC. We expanded our analysis to a nonoverlapping cohort of 205 patients and determined that DDR1 expression was upregulated in 126/205 PDAC specimens. Elevated DDR1 expression has also been reported in: (i) $52.2 \%$ of hepatocellular carcinoma samples [11]; (ii) $61.0 \%$ of non-small cell lung cancer [21]; and (iii) $63 \%$ of serous ovarian cancer tissues [13]. The overexpression of DDR1 in these different human cancers support the hypothesis that DDR1 may impact tumorigenesis and/or tumor progression.
Previous studies indicated that DDR1 could promote tumor progression by inducing cell adhesion and differentiation, which might be due to: (i) coexpressing with adhesion molecules [22]; (ii) promoting epithelial-mesenchymal transition (EMT) [21, 23]; (iii) participating in functional interaction of Notch1 and NF-кB pathway [24, 25]. Furthermore, survival analysis in our study revealed that PDAC patients with high DDR1 expression levels had significantly shorter survival times than those with low expression levels. Univariate analyses showed that increased DDR1 expression was significantly associated with the overall survival rate in PDAC patients. Multivariate analysis demonstrated that DDR1 expression, together with some traditional prognostic factors, such as age, $\mathrm{N}$ stage and liver metastasis, were independent risk factors in the prognosis of PDAC patients. These results suggested that DDR1 may represent a novel prognostic marker for PDAC patients.

The precise molecular mechanisms through which DDR1's impacts on tumor development and differentiation have yet to be elucidated. DDR1 presents 15 tyrosine residues in cell's cytoplasmic regions, which are potential sites for phosphorylation and receptor activation by different types of collagens $[6,12,26]$. It has been shown that over-expression of DDR1 increased the migration and invasion of hepatoma cells in vitro, which implicated a role of DDR1 in tumor progression and metastatic dissemination [27]. Reduced or absent DDR1 expression in vivo leads to defects in placental implantation and development of mammary gland [28], while Miao et al.[21] demonstrated that DDR1 expression promoted epithelial-tomesenchymal transition and contributed to non-smallcell lung cancer cells migration and invasion. The signaling

Table 2 Univariate and multivariate analyses of prognostic parameters for survival in patients with pancreatic ductal adenocarcinoma (PDAC)

\begin{tabular}{|c|c|c|c|c|c|c|}
\hline \multirow[b]{2}{*}{ Prognostic parameter } & \multicolumn{3}{|c|}{ Univariate analysis } & \multicolumn{3}{|c|}{ Multivariate analysis } \\
\hline & $\overline{R R}$ & $95 \% \mathrm{Cl}$ & $P$ value & 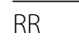 & $95 \% \mathrm{Cl}$ & $P$ value \\
\hline DDR1 (low vs.high) & 0.613 & $0.439-0.857$ & $0.004^{\mathrm{a}}$ & 0.643 & $0.457-0.904$ & $0.011^{\mathrm{a}}$ \\
\hline Age (<65 vs. $\geq 65)$ & 1.460 & $1.066-2.000$ & $0.019^{a}$ & 1.533 & $1.107-2.122$ & $0.010^{\mathrm{a}}$ \\
\hline Gender (male vs. female) & 0.765 & $0.552-1.058$ & 0.105 & & & \\
\hline Tumor location (head vs. body/tail) & 0.999 & $0.713-1.399$ & 0.996 & & & \\
\hline Size $(\leq 2 \mathrm{~cm}$ vs. $>2 \mathrm{~cm})$ & 1.641 & $0.978-2.754$ & 0.061 & & & \\
\hline Tumor differentiation (well vs. moderate/poor) & 2.125 & $0.938-4.813$ & 0.071 & & & \\
\hline T classification (T1 vs. T2 vs. T3 vs. T4) & 1.104 & $0.871-1.400$ & 0.412 & & & \\
\hline AJCC stage (I vs. I| vs. III vs. IV) & 1.260 & $0.992-1.600$ & 0.059 & & & \\
\hline N classification (absent vs. present) & 1.811 & $1.290-2.541$ & $0.001^{\mathrm{a}}$ & 1.769 & $1.242-2.519$ & $0.002^{\mathrm{a}}$ \\
\hline Liver metastasis (absent vs. present) & 3.299 & $1.746-6.235$ & $0.000^{\mathrm{a}}$ & 2.748 & $1.419-5.320$ & $0.003^{\mathrm{a}}$ \\
\hline Vascular invasion (absent vs. present) & 1.492 & $0.955-2.334$ & 0.079 & & & \\
\hline
\end{tabular}

HR hazard ratio, $\mathrm{Cl}$ confidence interval

AJCC staging is according to the $7^{\text {th }}$ edition of the American Joint Committee on Cancer (AJCC) staging system [29]

${ }^{\text {a }}$ Statistical significant $(P<0.05)$ 
pathways contributed by DDR1 upon cell-matrix interaction remain elusive and need further investigation.

In conclusion, this study demonstrated that DDR1 might serve as a novel prognostic biomarker in PDAC. Importantly, the molecular mechanisms underlying the relationship described above require clarification. Further studies are needed to investigate the molecular pathways involved in the regulation of DDR1, to improve our understanding and explore the possible therapies.

\section{Competing interests}

The authors declare that they have no competing interests.

\section{Authors' contributions}

Rong Hua and Yong-Wei Sun designed the research; Xueliang Fu, Dejun Liu, Jiao Li and Junfeng Zhang analysed the data; Yanmiao Huo, Minwei Yang, Wei Liu and Jianyu Yang performed the research and wrote the paper. All authors read and approved the final manuscript.

\section{Acknowledgements}

This study was supported by the National Natural Science Foundation of China (Grant No. 81401931) and the Young Medical Doctors Training and Funding Project of Shanghai Municipal Commission of Health and Family Planning. The funders had no role in study design, data collection and analysis, decision to publish, or preparation of the manuscript.

Received: 18 May 2015 Accepted: 6 August 2015

Published online: 22 August 2015

\section{References}

1. Stathis A, Moore MJ. Advanced pancreatic carcinoma: current treatment and future challenges. Nat Rev Clin Oncol. 2010;7(3):163-72. doi:10.1038/ nrclinonc.2009.236.

2. Hariharan D, Saied A, Kocher HM. Analysis of mortality rates for pancreatic cancer across the world. HPB Off J Int Hepato Pancreato Biliary Assoc. 2008;10(1):58-62. doi:10.1080/13651820701883148.

3. Jiang SH, He P, Ma MZ, Wang Y, Li RK, Fang F, et al. PNMA1 promotes cell growth in human pancreatic ductal adenocarcinoma. Int J Clin Exp Pathol. 2014;7(7):3827-35.

4. Siegel R, Ma J, Zou Z, Jemal A. Cancer statistics, 2014. CA Cancer J Clin. 2014;64(1):9-29. doi:10.3322/caac.21208.

5. Remmers N, Bailey JM, Mohr AM, Hollingsworth MA. Molecular pathology of early pancreatic cancer. Cancer Biomarkers Section Dis Markers. 2010:9(1-6):421-40. doi:10.3233/CBM-2011-0168.

6. Vogel WF, Abdulhussein R, Ford CE. Sensing extracellular matrix: an update on discoidin domain receptor function. Cell Signal. 2006;18(8):1108-16. doi:10.1016/j.cellsig.2006.02.012

7. Borza CM, Pozzi A. Discoidin domain receptors in disease. Matrix Biol J Int Soc Matrix Biol. 2014;34:185-92. doi:10.1016/j.matbio.2013.12.002.

8. Ferri N, Carragher NO, Raines EW. Role of discoidin domain receptors 1 and 2 in human smooth muscle cell-mediated collagen remodeling: potential implications in atherosclerosis and lymphangioleiomyomatosis. Am J Pathol. 2004;164(5):1575-85. doi:10.1016/50002-9440(10)63716-9.

9. Valencia K, Ormazabal C, Zandueta C, Luis-Ravelo D, Anton I, Pajares MJ, et al. Inhibition of collagen receptor discoidin domain receptor-1 (DDR1) reduces cell survival, homing, and colonization in lung cancer bone metastasis. Clin Cancer Res Off J Am Assoc Cancer Res. 2012;18(4):969-80. doi:10.1158/1078-0432.CCR-11-1686.

10. Yang SH, Baek HA, Lee HJ, Park HS, Jang KY, Kang MJ, et al. Discoidin domain receptor 1 is associated with poor prognosis of non-small cell lung carcinomas. Oncol Rep. 2010;24(2):311-9.

11. Shen Q, Cicinnati VR, Zhang X, lacob S, Weber F, Sotiropoulos GC, et al. Role of microRNA-199a-5p and discoidin domain receptor 1 in human hepatocellular carcinoma invasion. Mol Cancer. 2010;9:227. doi:10.1186/ 1476-4598-9-227.

12. Castro-Sanchez L, Soto-Guzman A, Guaderrama-Diaz M, Cortes-Reynosa P, Salazar EP. Role of DDR1 in the gelatinases secretion induced by native type IV collagen in MDA-MB-231 breast cancer cells. Clin Exp Metastasis. 2011;28(5):463-77. doi:10.1007/s10585-011-9385-9.
13. Quan J, Yahata T, Adachi S, Yoshihara K, Tanaka K. Identification of receptor tyrosine kinase, discoidin domain receptor 1 (DDR1), as a potential biomarker for serous ovarian cancer. Int J Mol Sci. 2011;12(2):971-82. doi:10.3390/ijms12020971.

14. Von Hoff DD, Ervin T, Arena FP, Chiorean EG, Infante J, Moore M, et al. Increased survival in pancreatic cancer with nab-paclitaxel plus gemcitabine. N Engl J Med. 2013;369(18):1691-703. doi:10.1056/NEJMoa1304369.

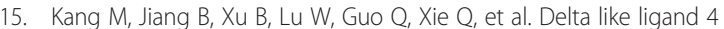
induces impaired chemo-drug delivery and enhanced chemoresistance in pancreatic cancer. Cancer Lett. 2013;330(1):11-21. doi:10.1016/ j.canlet.2012.11.015.

16. Yang JY, Sun YW, Liu DJ, Zhang JF, Li J, Hua R. MicroRNAs in stool samples as potential screening biomarkers for pancreatic ductal adenocarcinoma cancer. Am J Cancer Res. 2014;4(6):663-73.

17. Liang X, Sun S, Zhang X, Wu H, Tao W, Liu T et al. Expression of ribosome-binding protein 1 correlates with shorter survival in Her-2 positive breast cancer. Cancer science. 2015. doi:10.1111/cas.12666

18. Li J, Yang XM, Wang YH, Feng MX, Liu XJ, Zhang YL, et al. Monoamine oxidase A suppresses hepatocellular carcinoma metastasis by inhibiting the adrenergic system and its transactivation of EGFR signaling. J Hepatol. 2014;60(6):1225-34. doi:10.1016/.j.jhep.2014.02.025.

19. Ling H, Pickard K, Ivan C, Isella C, Ikuo M, Mitter R et al. The clinical and biological significance of MIR-224 expression in colorectal cancer metastasis. Gut. 2015. doi:10.1136/gutjnl-2015-309372

20. Buchholz M, Braun M, Heidenblut A, Kestler HA, Kloppel G, Schmiegel W, et al. Transcriptome analysis of microdissected pancreatic intraepithelial neoplastic lesions. Oncogene. 2005;24(44):6626-36. doi:10.1038/ sj.onc.1208804.

21. Miao LY, Zhu SH, Wang YS, Li Y, Ding JJ, Dai JH, et al. Discoidin domain receptor 1 is associated with poor prognosis of non-small cell lung cancer and promotes cell invasion via epithelial-to-mesenchymal transition. Med Oncol. 2013;30(3):626. doi:10.1007/S12032-013-0626-4. doi:Unsp.

22. Heinzelmann-Schwarz VA, Gardiner-Garden M, Henshall SM, Scurry J, Scolyer RA, Davies MJ, et al. Overexpression of the cell adhesion molecules DDR1, Claudin 3, and Ep-CAM in metaplastic ovarian epithelium and ovarian cancer. Clin Cancer Res Off J Am Assoc Cancer Res. 2004;10(13):4427-36. doi:10.1158/1078-0432.CCR-04-0073.

23. Maeyama M, Koga H, Selvendiran K, Yanagimoto C, Hanada S, Taniguchi E, et al. Switching in discoid domain receptor expressions in SLUG-induced epithelial-mesenchymal transition. Cancer. 2008;113(10):2823-31. doi:10.1002/cncr.23900.

24. Lu KK, Trcka D, Bendeck MP. Collagen stimulates discoidin domain receptor 1-mediated migration of smooth muscle cells through Src. Cardiovas Pathol Off J Soc Cardiovas Pathol. 2011;20(2):71-6. doi:10.1016/ j.carpath.2009.12.006

25. Curat CA, Vogel WF. Discoidin domain receptor 1 controls growth and adhesion of mesangial cells. J Am Soc Nephrol JASN. 2002;13(11):2648-56.

26. Vogel W. Discoidin domain receptors: structural relations and functional implications. FASEB J Off Publ Federation Am Soc Exp Biol. 1999;13(Suppl):S77-82.

27. Park HS, Kim KR, Lee HJ, Choi HN, Kim DK, Kim BT, et al. Overexpression of discoidin domain receptor 1 increases the migration and invasion of hepatocellular carcinoma cells in association with matrix metalloproteinase. Oncol Rep. 2007;18(6):1435-41.

28. Vogel WF, Aszodi A, Alves F, Pawson T. Discoidin domain receptor 1 tyrosine kinase has an essential role in mammary gland development. Mol Cell Biol. 2001;21(8):2906-17. doi:10.1128/MCB.21.8.2906-2917.2001.

29. McIntyre CA, Winter JM. Diagnostic evaluation and staging of pancreatic ductal adenocarcinoma. Semin Oncol. 2015;42(1):19-27. doi:10.1053/ j.seminoncol.2014.12.003. 